\title{
Front Matter: Volume 8629
}

, "Front Matter: Volume 8629," Proc. SPIE 8629, Silicon Photonics VIII, 862901 (14 March 2013); doi: 10.1117/12.2021448

SPIE. Event: SPIE OPTO, 2013, San Francisco, California, United States 


\section{PROCEEDINGS OF SPIE}

\section{Silicon Photonics VIII}

Joel Kubby

Graham T. Reed

Editors

4-6 February 2013

San Francisco, California, United States

Sponsored and Published by

SPIE 
The papers included in this volume were part of the technical conference cited on the cover and title page. Papers were selected and subject to review by the editors and conference program committee. Some conference presentations may not be available for publication. The papers published in these proceedings reflect the work and thoughts of the authors and are published herein as submitted. The publisher is not responsible for the validity of the information or for any outcomes resulting from reliance thereon.

Please use the following format to cite material from this book:

Author(s), "Title of Paper," in Silicon Photonics VIII, edited by Joel Kubby, Graham T. Reed, Proceedings of SPIE Vol. 8629 (SPIE, Bellingham, WA, 2013) Article CID Number.

ISSN: 0277-786X

ISBN: 9780819493989

Published by

SPIE

P.O. Box 10, Bellingham, Washington 98227-0010 USA

Telephone +1 3606763290 (Pacific Time) · Fax +1 3606471445

SPIE.org

Copyright (C) 2013, Society of Photo-Optical Instrumentation Engineers.

Copying of material in this book for internal or personal use, or for the internal or personal use of specific clients, beyond the fair use provisions granted by the U.S. Copyright Law is authorized by SPIE subject to payment of copying fees. The Transactional Reporting Service base fee for this volume is $\$ 18.00$ per article (or portion thereof), which should be paid directly to the Copyright Clearance Center (CCC), 222 Rosewood Drive, Danvers, MA 01923. Payment may also be made electronically through $\mathrm{CCC}$ Online at copyright.com. Other copying for republication, resale, advertising or promotion, or any form of systematic or multiple reproduction of any material in this book is prohibited except with permission in writing from the publisher. The CCC fee code is $0277-786 \mathrm{X} / 13 / \$ 18.00$.

Printed in the United States of America.

Publication of record for individual papers is online in the SPIE Digital Library.

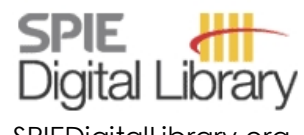

SPIEDigitalLibrary.org

Paper Numbering: Proceedings of SPIE follow an e-First publication model, with papers published first online and then in print and on CD-ROM. Papers are published as they are submitted and meet publication criteria. A unique, consistent, permanent citation identifier (CID) number is assigned to each article at the time of the first publication. Utilization of CIDs allows articles to be fully citable as soon as they are published online, and connects the same identifier to all online, print, and electronic versions of the publication. SPIE uses a six-digit CID article numbering system in which:

- The first four digits correspond to the SPIE volume number.

- The last two digits indicate publication order within the volume using a Base 36 numbering

system employing both numerals and letters. These two-number sets start with 00, 01, 02, 03, 04, $05,06,07,08,09,0 A, 0 B \ldots$. OZ, followed by 10-1Z, 20-2Z, etc.

The CID Number appears on each page of the manuscript. The complete citation is used on the first page, and an abbreviated version on subsequent pages. Numbers in the index correspond to the last two digits of the six-digit CID Number. 


\section{Contents}

ix Conference Committee

xi Light in a twist: optical angular momentum (Plenary Paper) [8637-2]

M. J. Padgett, Univ. of Glasgow (United Kingdom)

\section{PLENARY SESSION}

862902 Group IV photonics for the mid infrared (Plenary Paper) [8629-1]

R. Soref, The Univ. of Massachusetts at Boston (United States)

LAB-ON-A-CHIP I

862904 Silicon photonics for functional on-chip optical tweezers devices and circuits (Invited Paper) [8629-3]

H. Cai, Univ. of California, Santa Cruz (United States); J. Wang, A. W. Poon, Hong Kong Univ. of Science and Technology (Hong Kong, China)

862905 Ring resonator based SOI biosensors (Invited Paper) [8629-4]

P. Bienstman, S. Werquin, C. Lerma Arce, Ghent Univ. (Belgium); D. Witters, R. Puers,

J. Lammertyn, Katholieke Univ. Leuven (Belgium); T. Claes, E. Hallynck, J.-W. Hoste,

D. Martens, Ghent Univ. (Belgium)

862906 Monolithic silicon interferometric optoelectronic devices for label-free multi-analyte biosensing applications (Invited Paper) [8629-5]

K. Misiakos, E. Makarona, I. Raptis, A. Salapatas, A. Psarouli, S. Kakabakos, P. Petrou, National Ctr. for Scientific Research Demokritos (Greece); M. Hoekman, LioniX B.V. (Netherlands); R. Stoffer, PhoeniX B.V. (Netherlands); K. Tukkiniemi, VTT Tietotie (Finland); G. Jobst, Jobst Technologies GmbH (Germany)

\section{LAB-ON-A-CHIP II}

862907 Bioinspired optofluidic lasers for DNA and protein detection (Invited Paper) [8629-6] X. Zhang, Univ. of Michigan (United States) and Fudan Univ. (China); Q. Chen, M. Ritt, S. Sivaramakrishnan, X. Fan, Univ. of Michigan (United States)

862909 Label-free silicon photonic biosensors for use in clinical diagnostics (Invited Paper) [8629-8] S. Talebi Fard, S. M. Grist, V. Donzella, The Univ. of British Columbia (Canada); S. A. Schmidt, Univ. of Washington (United States); J. Flueckiger, X. Wang, W. Shi, The Univ. of British Columbia (Canada); A. Millspaugh, Univ. of Washington (United States); M. Webb, The Univ. of British Columbia (Canada); D. M. Ratner, Univ. of Washington (United States); K. C. Cheung, L. Chrostowski, The Univ. of British Columbia (Canada) 
8629 OB Ultracompact polarization diversity components for future large-scale photonic integrated circuits on silicon (Invited Paper) [8629-10]

D. Dai, Zhejiang Univ. (China)

8629 OC Deeply etched MMI-based components on $\mathbf{4} \boldsymbol{\mu m}$ thick SOI for SOA-based optical RAM cell circuits [8629-11]

M. Cherchi, S. Ylinen, M. Harjanne, M. Kapulainen, T. Aalto, VTT Technical Research Ctr. of Finland (Finland); G. T. Kanellos, Informatics and Telematics Institute (Greece); D. Fitsios, Informatics and Telematics Institute (Greece) and Aristotle Univ. of Thessaloniki (Greece); N. Pleros, Informatics and Telematics Institute (Greece)

8629 OD Bend-size reduction on the SOI rib waveguide platform [8629-12] T. Aalto, M. Cherchi, M. Harjanne, S. Ylinen, M. Kapulainen, VTT Technical Research Ctr. of Finland (Finland)

8629 OE Fabrication of low-loss silicon nanophotonic waveguide for photonic device integration [8629-13]

D. K. T. Ng, K.-P. Lim, Q. Wang, J. Pu, K. Tang, Y. Lai, C.-W. Lee, Data Storage Institute (Singapore); S.-T. Ho, Data Storage Institute (Singapore) and Northwestern Univ. (United States)

\section{WAVEGUIDES II}

8629 OF Integration of silicon photonics into electronic processes (Invited Paper) [8629-14] J. S. Orcutt, R. J. Ram, V. Stojanović, Massachusetts Institute of Technology (United States)

8629 OG Ion beam irradiation induced fabrication of vertical coupling photonic structures [8629-15] H. D. Liang, V. S. Kumar, J. F. WU, M. B. H. Breese, National Univ. of Singapore (Singapore)

$8629 \mathrm{OH}$ Highly efficient DBR in silicon waveguides with eleventh order diffraction [8629-16]

S. Harish, D. Venkitesh, B. K. Das, I Indian Institute of Technology Madras (India)

8629 Ol Experimental demonstration of 2D photonic crystal, triangular lattice, small angle, low loss Y-Splitter at microwave frequencies [8629-17]

D. Kaushal, R. C. Gauthier, Carleton Univ. (Canada)

8629 0J Mid-infrared photonics devices in SOI (Invited Paper) [8629-18]

G. Z. Mashanovich, M. Nedeljkovic, Univ. of Southampton (United Kingdom);

M. M. Milošević, Univ. of Surrey (United Kingdom); Y. Hu, T. M. Ben Masaud, E. Jaberansary,

X. Chen, Univ. of Southampton (United Kingdom); M. Strain, M. Sorel, Univ. of Glasgow (United Kingdom); A. C. Peacock, H. M. H. Chong, G. T. Reed, Univ. of Southampton (United Kingdom)

8629 OK Silicon slot waveguides and their rigorous characterization [8629-19]

B. M. A. Rahman, D. M. H. Leung, N. Kejalakshmy, L. To, City Univ. London (United Kingdom) 
SILICON PHOTONICS MEETS EO-POLYMERS: JOINT KEYNOTE SESSION WITH CONFERENCES 8622, 8624, AND 8629

8629 OL How will photonic integrated circuits develop? (Keynote Paper) [8629-20]

M. W. Haney, Univ. of Delaware (United States)

PHOTONIC CRYSTALS AND WIRES

8629 OM Integrated tunable silicon photonic devices for optical filter and delay applications (Invited Paper) [8629-21]

L. Zhou, X. Sun, J. Xie, L. Lu, Z. Zou, J. Chen, Shanghai Jiao Tong Univ. (China)

8629 ON Reconfigurable 3D photonic crystal structures [8629-22]

R. C. Gauthier, Carleton Univ. (Canada)

MODULATORS AND DETECTORS I

$862900 \quad \lambda$-size silicon-based modulator (Invited Paper) [8629-23]

V. J. Sorger, The George Washington Univ. (United States) and Univ. of California Berkeley

(United States); N. D. Lanzillotti-Kimura, R.-M. Ma, Univ. of California Berkeley (United States);

C. Huang, Z. Li, The George Washington Univ. (United States); X. Zhang, Univ. of California Berkeley (United States)

8629 OP Silicon-organic hybrid devices (Invited Paper) [8629-24]

L. Alloatti, D. Korn, J. Pfeifle, R. Palmer, S. Koeber, M. Baier, R. Schmogrow, S. Diebold, P. Pahl, T. Zwick, Karlsruhe Institute of Technology (Germany); H. YU, W. Bogaerts, R. Baets, Ghent Univ. (Belgium); M. Fournier, J. Fedeli, CEA-LETI, Minatec (France); R. Dinu, Gigoptix Inc. (United States); C. Koos, W. Freude, J. Leuthold, Karlsruhe Institute of Technology (Germany)

$86290 Q \quad 40 \mathrm{~Gb} / \mathrm{s}$ low-loss self-aligned silicon optical modulator [8629-25]

M. Ziebell, D. Marris-Morini, G. Rasigade, Institut d'Électronique Fondamentale, Univ. Paris Sud XI (France); J.-M. Fédéli, CEA-LETI, Minatec (France); E. Cassan, L. Vivien, Institut d'Électronique Fondamentale, Univ. Paris Sud XI (France)

8629 OR Waveguide integrated silicon avalanche photodetectors [8629-26]

J. J. Ackert, E. Huante-Ceron, K. J. Murray, McMaster Univ. (Canada); P. E. Jessop, Wilfrid Laurier Univ. (Canada); A. P. Knights, McMaster Univ. (Canada)

8629 OS Accurate high-speed eye diagram simulation of silicon-based modulators [8629-27] C. E. Png, V. Dixit, S. T. Lim, E.-P. Li, Institute of High Performance Computing (Singapore)

8629 OT Interferometric switching in CROW based reconfigurable optical device for routing application [8629-28]

M. Mancinelli, P. Bettotti, Univ. of Trento (Italy); J. Fedeli, CEA-LETI, Minatec (France);

L. Pavesi, Univ. of Tento (Italy) 
8629 OU Low-loss high-speed silicon Mach-Zehnder modulator for optical-fiber telecommunications (Invited Paper) [8629-29]

K. Ogawa, K. Goi, H. Kusaka, Y. Terada, Fujikura Ltd. (Japan); T.-Y. Liow, X. Tu, G.-Q. Lo, D.-L. Kwong, Institute of Microelectronics (Singapore); V. Dixit, S. T. Lim, C. E. Png, Institute of High Performance Computing (Singapore)

\section{MODULATORS AND DETECTORS III}

8629 0X Optical modulation using the silicon platform (Invited Paper) [8629-32]

F. Y. Gardes, D. Thomson, Y. Hu, G. T. Reed, Univ. of Surrey (United Kingdom); L. O'Faolain, K. Debnath, T. F. Krauss, Univ. of St. Andrews (United Kingdom); J. M. Fedeli, L. Lever, R. W. Kelsall, Z. Ikonic, CEA-LETI, Minatec (United Kingdom); X.-C. Liu, M. Myronov, D. R. Leadley, Institute of Microwaves and Photonics (United Kingdom)

$86290 Z$ A low power electro-optic polymer clad Mach-Zehnder modulator for high speed optical interconnects [8629-34]

B. Block, S. Liff, M. Kobrinsky, M. Reshotko, R. Tseng, I. Ban, P. Chang, Intel Corp. (United States)

862910 Polarization-independent and dispersion-free integrated optical MZI in SOI substrate for DWDM applications [8629-35]

U. Karthik, B. K. Das, Indian Institute of Technology Madras (India)

\section{EMITTERS AND LASERS I}

862911 Hybrid silicon free-space source with integrated beam steering (Invited Paper) [8629-36]

J. K. Doylend, M. J. R. Heck, J. T. Bovington, J. D. Peters, M. L. Davenport, L. A. Coldren,

J. E. Bowers, Univ. of California, Santa Barbara (United States)

862913 A novel type of ultra-compact lateral-current-injection III/V photonic device integrated on SOI for electronic-photonic chip application [8629-38]

J. Pu, Q. Wang, Data Storage Institute (Singapore); S. Ho, Data Storage Institute

(Singapore) and Northwestern Univ. (United States)

862914 Role of electron and hole transport processes in conductivity and light emission of silicon nanocrystals field-effect transistors [8629-39]

L. Cattoni, A. Tengattini, A. Anopchenko, Univ. of Trento (Italy); J. M. Ramirez, F. Ferrarese Lupi, Y. Berencen, B. Garrido, Univ. de Barcelona (Spain); J.-M. Fedeli, CEA-LETI, Minatec (France); L. Pavesi, Univ. of Trento (Italy)

\section{EMITTERS AND LASERS II}

862916 Experimental demonstration of a novel heterogeneously integrated III-V on Si microlaser [8629-41]

Y. De Koninck, Ghent Univ. (Belgium); F. Raineri, A. Bazin, R. Raj, Lab. de Photonique et de Nanostructure, CNRS (France); G. Roelkens, R. Baets, Ghent Univ. (Belgium) 
862918 Room temperature electrically pumped silicon nano-light source at telecommunication wavelengths [8629-43]

A. Shakoor, Univ. of St. Andrews (United Kingdom); R. Lo Savio, Univ. di Pavia (Italy);

P. Cardile, Univ. di Catania (Italy); S. L. Portalupi, D. Gerace, Univ. di Pavia (Italy); K. Welna, Univ. of St. Andrews (United Kingdom); S. Boninelli, G. Franzò, F. Priolo, Univ. di Catania (Italy); T. F. Krauss, Univ. of St Andrews (United Kingdom); M. Galli, Univ. di Pavia (Italy);

L. O'Faolain, Univ. of St Andrews (United Kingdom)

POSTER SESSION

86291 A Responsivity measurements of N-on-P and P-on-N silicon photomultipliers in the continuous wave regime [8629-45]

G. Adamo, D. Agrò, S. Stivala, A. Parisi, C. Giaconia, A. Busacca, Univ. degli Studi di Palermo (Italy); M. C. Mazzillo, D. Sanfilippo, P. G. Fallica, STMicroelectronics (Italy)

8629 1C Anomalous localization modes in Bragg-grating based on high index-difference waveguide [8629-47]

T. Kita, K. Uchijima, H. Yamada, Tohoku Univ. (Japan)

8629 ID All-optical single resonance control using a silicon-based ring-assisted Mach-Zehnder interferometer [8629-48]

Y. Xiong, W. N. Ye, Carleton Univ. (Canada)

8629 IE Silicon nanomembrane based photonic crystal waveguide true-time-delay lines on a glass substrate [8629-49]

H. Subbaraman, Omega Optics, Inc. (United States); X. Xu, C.-Y. Lin, The Univ. of Texas at Austin (United States); A. Hosseini, Omega Optics, Inc. (United States); R. T. Chen, The Univ. of Texas at Austin (United States)

8629 IF Low power consumption silicon photonics tuning filters based on compound microring resonators [8629-50]

C. Vázquez, P. Contreras, Univ. Carlos III de Madrid (Spain); S. Vargas, Univ. Tecnológica de Panamá (Panama)

8629 1G Large area transferred silicon nanomembrane photonic devices on unconventional substrates [8629-51]

X. XU, The Univ. of Texas at Austin (United States); H. Subbaraman, A. Hosseini, Omega Optics, Inc. (United States); D. Kwong, R. T. Chen, The Univ. of Texas at Austin (United States)

8629 1H Silicon nanocrystal density effects in sensitizing erbium atoms [8629-52]

M. Q. Huda, Bangladesh Univ. of Engineering and Technology (Bangladesh)

8629 i A highly accurate engineering of silicon integrated microring resonators based on the nanofabrication technique [8629-54]

M. Erdmanis, L. Karvonen, A. Säynätjoki, Aalto Univ. (Finland); S. Honkanen, Univ. of Eastern Finland (Finland); I. Tittonen, Aalto Univ. (Finland)

Author Index 
Proc. of SPIE Vol. $8629862901-8$

Downloaded From: https://www.spiedigitallibrary.org/conference-proceedings-of-spie on 26 Apr 2023 Terms of Use: https://www.spiedigitallibrary.org/terms-of-use 


\section{Conference Committee}

Symposium Chair

David L. Andrews, University of East Anglia Norwich (United Kingdom)

Symposium Cochairs

Alexei L. Glebov, OptiGrate Corporation (United States)

Klaus P. Streubel, OSRAM GmbH (Germany)

Program Track Chair

Yakov Sidorin, Quarles Brady LLP (United States)

Conference Chairs

Joel Kubby, University of California, Santa Cruz (United States)

Graham T. Reed, University of Southampton (United Kingdom)

Conference Program Committee

Laurence W. Cahill, La Trobe University (Australia)

Philippe M. Fauchet, Vanderbilt University (United States)

L. Cary Gunn, Genalyte, Inc. (United States)

Siegfried Janz, National Research Council Canada (Canada)

Andrew P. Knights, McMaster University (Canada)

Laura Maria Lechuga, Catalan Institute of Nanoscience and Nanotechnology (Spain)

Sebania Libertino, Istituto per la Microelettronica e Microsistemi (Italy)

Goran Z. Mashanovich, University of Southampton (United Kingdom)

Ching Eng Jason Png, A*STAR Institute of High Performance

Computing (Singapore)

Andrew W. Poon, Hong Kong University of Science and Technology

(Hong Kong, China)

Haisheng Rong, Intel Corporation (United States)

Holger Schmidt, University of California, Santa Cruz (United States)

Danxia Xu, National Research Council Canada (Canada)

Zhiping Zhou, Peking University (China) 
Plenary Session

David L. Andrews, University of East Anglia Norwich (United Kingdom)

Alexei L. Glebov, OptiGrate Corporation (United States)

1 Lab-on-a-Chip I

Andrew W. Poon, Hong Kong University of Science and Technology (Hong Kong, China)

2 Lab-on-a-Chip II

Andrew W. Poon, Hong Kong University of Science and Technology (Hong Kong, China)

3 Waveguides I

Zhiping Zhou, Peking University (China)

4 Waveguides II

Zhiping Zhou, Peking University (China)

5 Silicon Photonics Meets EO-Polymers: Joint Keynote Session with Conferences 8622, 8624, and 8629

Robert A. Norwood, University of Arizona (United States)

6 Photonic Crystals and Wires

Graham T. Reed, University of Southampton (United Kingdom)

7 Modulators and Detectors I

Graham T. Reed, University of Southampton (United Kingdom)

8 Modulators and Detectors II

Andrew P. Knights, McMaster University (Canada)

9 Modulators and Detectors III

Andrew P. Knights, McMaster University (Canada)

10 Emitters and Lasers I

Ching Eng Jason Png, A*STAR Institute of High Performance Computing (Singapore)

11 Emitters and Lasers II

Ching Eng Jason Png, A*STAR Institute of High Performance Computing (Singapore) 